\title{
In this Issue of Worm
}

Elaine L. Ellerton

Landes Bioscience; Austin, TX USA

\section{Research Papers: Choosy Females}

Caenorhabditis elegans does not require a male in order to reproduce; it has the ability to reproduce asexually. Caenorhabditis remanei, on the other hand, must copulate with a male in order to reproduce. Male C. remanei are notorious philanderers and even will even stray outside their own species, but do the females share such a wondering eye? Markert and García tested this theory on virgin C. remanei. Did they have any mate selection bias? The authors found that females did indeed show a preference, but only for males recently or currently copulating and only of the same species. They suggest that this behavior is pheromone based. (Fig. 1)

\section{Electric Microfluidics Uncover Neuronal Behavior}

Understanding the neuronal basis behind certain behaviors is a difficult task and novel ways to speed up this process are always welcome. In this research paper, Salam et al. provide us with just that. Using C. elegans, the authors use an electric field within a microfluidic device to probe neuronal activity. With the help of mutant strains and worms treated with neurotoxins, the group was able to determine that this microfluidic setup could reliably detect neuronal abnormalities thus making this platform a useful screening device for uncovering neuronal function. (Fig. 2)

\section{Mini-review: Nematode Neuromodulation}

Vasopressin and oxytocin are two important neuropeptide hormones highly involved in the regulation of animal physiology and behavior. How exactly these hormones exert their effects is not known. In this mini-review, Beets, et al. describe recently discovered C. elegans vasopressin/ oxytocin related hormones and how they compare with similar hormones seen in the mammalian brain. Studying this hormonal system in the nematode will hopefully lead to a deeper understanding of the mechanisms underpinning these two hormones. (Fig. 3)

\section{Commentaries: Histone 1 and Heterochromatin Protein 1 Keep it Specific}

The correct transcription of Hox genes is pretty important if you want limb development to occur in the right places. Once again, C. elegans provides a good model to study the inappropriate expression of Hox genes. In this commentary, Monika Jedrusik-Bode comments on recent findings that focus on the histone $\mathrm{H} 1$ and heterochromatin protein 1 and their selective mechanisms underlying Hox gene regulation.

\section{ROS Role in Cuticle Development}

C. elegans retain their shapely figures with a tough and flexible cuticle. This outercovering is comprised of cross-linked collagen molecules, a process that requires reactive oxygen species (ROS). Due to their highly reactive nature, the generation of ROS must be tightly controlled. In this commentary, Moribe and Mekada describe two molecules involved in ROS regulation, BLI-3 and TSP-15 and explain their roles in cuticle development.

\section{Apoptotic Proteins Promote Nerve Regeneration}

Laser ablation in $C$. elegans has proven itself invaluable in the quest to uncover

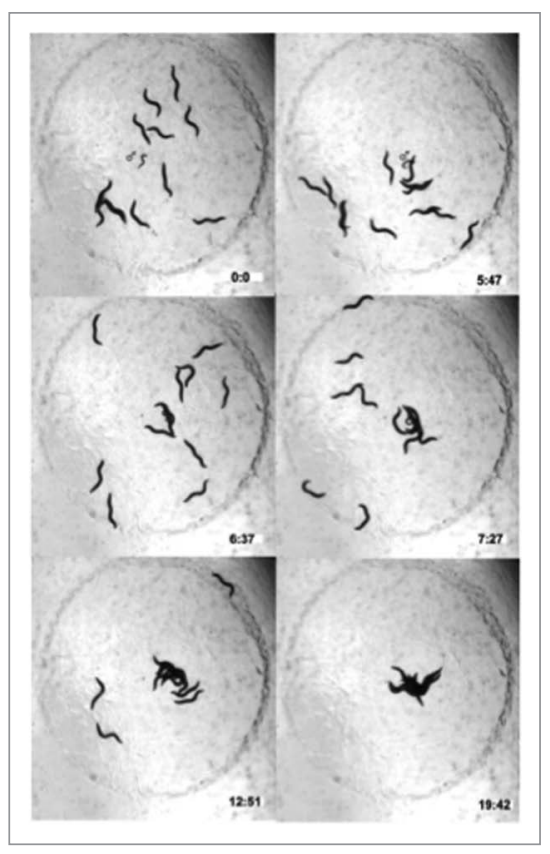

Figure 1. Figure 2 from Beets I, et al.

the mechanisms, or lack thereof, of regeneration in the central nervous system. On the heels of the discovery of DLK-1's involvement in regeneration and that it is highly conserved in other organisms, Reina, Driscoll and Gabel discuss their recent findings on two apoptotic proteins, CED-4/Apaf-1, along with the caspase, CED-3, which work upstream of DLK-1 to promote regeneration. The authors provide a working model and discuss mechanisms for the spatial regulation of activated CED-3. (Fig. 4)

\section{UTX-1 Drives Development without Catalytic Activity}

Recently, Vandamme, et al. found that $u t x-1$ is a critical gene for the development of C. elegans. However, using rescue experiments, the authors determined that the role of $u t x-1$ was not dependent 
on the enzymatic activity of this gene. In this commentary, possible mechanisms for the involvement of the UTX-1 complex in correct embryonic and post-embryonic development is discussed.

\section{Strict Regulation of Centrosome Duplication}

Cell division is a highly regulated process because if done incorrectly, problems linked to abnormal chromosomal number or even cancer, may arise. Therefore, it is critical that the control of centrosome duplication, an important part of mitosis, is highly regulated. Here, Nina Peel discusses the importance of protein degradation in regulating centrosome duplication, focusing specifically on the SCF ubiquitin ligase complex. (Fig. 5)

\section{Two Ways of Learning}

The molecular mechanisms that underpin the process of learning are not completely understood. Luckily, C. elegans provides us with a good model to studying this complicated phenomenon. In this commentary, Pereira and van der Kooy summarize a two input-two output stimuli system that is able to distinguish between two types of learning, associative and non-associative. They also explain how the intertwining of associative and nonassociative memory may be an artifact of the divergence between the two types of learning.

\section{Fold Properly for Function}

For a protein to function properly, it must have the correct tertiary structure, thus requiring proper folding. This takes place in the strictly controlled endoplasmic reticulum. One response that deals with misfolded proteins is the unfolded protein response (UPR). In this commentary, Sakaki and Kaufman review their recent findings garnered from a genetic screen that attempted to identify genes that interact with the UPR response. The authors discuss their findings and provide future avenues for study.

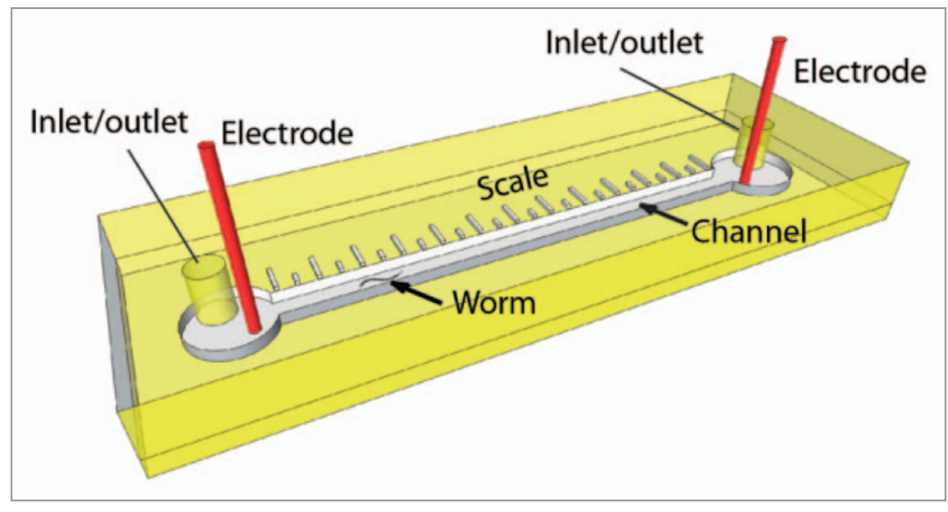

Figure 2. Part of Figure 1 from Reina $C P$, et al.

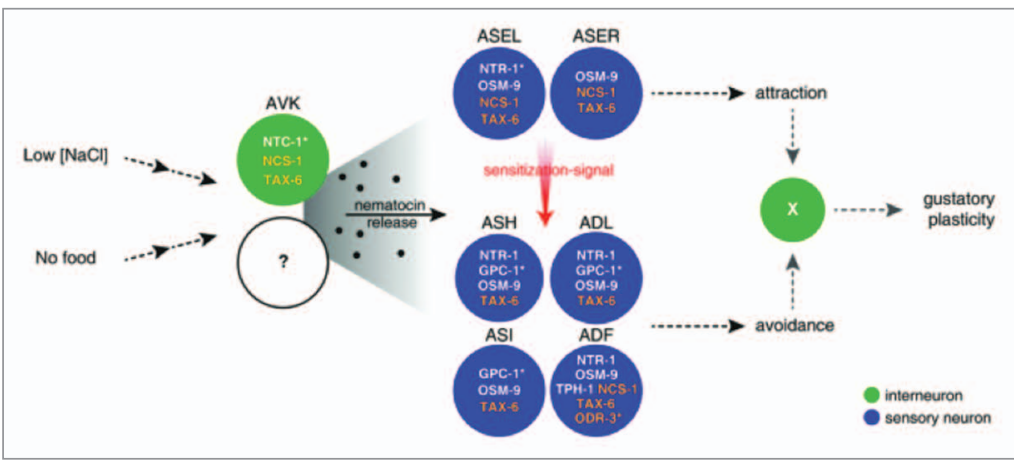

Figure 3. Figure 1 from Massirer, et al.

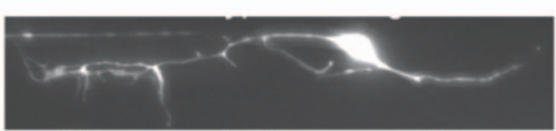

Neuronal Regeneration

Figure 4. Part of Figure 1 from Reina $C P$, et al.

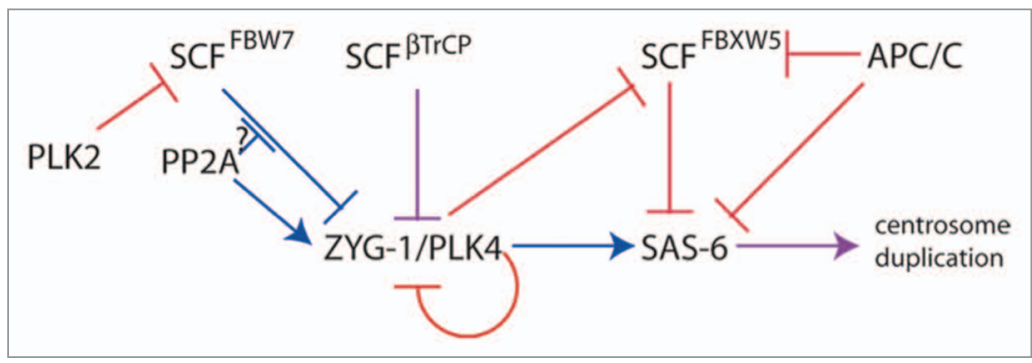

Figure 5. Figure 1 from Peel $\mathrm{N}$. 